\title{
Perfect state transfer on quotient graphs
}

\author{
Rachel Bachman \\ Clarkson University \\ Michael Landry \\ UC Berkeley
}

\author{
Eric Fredette \\ Clarkson University \\ Michael Opperman \\ Clarkson University
}

Andrew Tollefson

University of Nevada at Reno

\author{
Jessica Fuller \\ Seton Hall University \\ Christino Tamon* \\ Clarkson University
}

October 23, 2018

\begin{abstract}
We prove new results on perfect state transfer of quantum walks on quotient graphs. Since a graph $G$ has perfect state transfer if and only if its quotient $G / \pi$, under any equitable partition $\pi$, has perfect state transfer, we exhibit graphs with perfect state transfer between two vertices but which lack automorphism swapping them. This answers a question of Godsil (Discrete Mathematics 312(1):129-147, 2011). We also show that the Cartesian product of quotient graphs $\square_{k} G_{k} / \pi_{k}$ is isomorphic to the quotient graph $\square_{k} G_{k} / \pi$, for some equitable partition $\pi$. This provides an algebraic description of a construction due to Feder (Physical Review Letters 97, 180502, 2006) which is based on many-boson quantum walk.
\end{abstract}

Keywords: quantum walk, perfect state transfer, equitable partition, quotient graph.

\section{Introduction}

Perfect state transfer in continuous-time quantum walk on graphs has received considerable attention in quantum information and computation. This is in large part due to its potential applications in quantum information transmission over networks and its role in quantum computation. Recently, continuing ideas developed by Childs [9], Underwood and Feder [31] used perfect state transfer to show that continuous-time quantum walk is a universal computational model. The notion of perfect state transfer was introduced by Bose [7] in the context of information transfer on linear spin-chains. His original scheme can be generalized to arbitrary graphs (as described by Albanese et al. and Christandl et al. [1, 12, 11]) which we briefly outline in the following.

Given a weighted graph $G$ on $n$ vertices with adjacency matrix $A(G)$, we imagine a collection of $n$ qubits associated with each vertex of $G$ and arranged so that their interaction is governed by a Hamiltonian $\mathcal{H}_{G}$ which depends on the edge structure of $G$. Here, our collective Hilbert space is $\bigotimes_{u \in V} \mathbb{C}^{2}$ which is $2^{n}$-dimensional. Suppose an arbitrary one-qubit state $|\psi\rangle=\alpha_{0}|0\rangle+\alpha_{1}|1\rangle$ is located at vertex $a$ of $G$. Our goal is to move this state to vertex $b$. For simplicity, we depict $a$ as the leftmost qubit whereas $b$ is the rightmost qubit. The initial configuration has the qubit at vertex $a$ be in state $|\psi\rangle$ and the other qubits are in the $|0\rangle$ state, while the final configuration has

*Contact author: tino@clarkson.edu 
the qubit at vertex $b$ be in state $|\psi\rangle$ with the other qubits being in the $|0\rangle$ state:

$$
\begin{aligned}
|\mathrm{START}\rangle & =|\psi\rangle_{a} \otimes|0\rangle \otimes \ldots \otimes|0\rangle \otimes|0\rangle_{b}=\alpha_{0}|00 \ldots 00\rangle+\alpha_{1}|10 \ldots 00\rangle \\
|\mathrm{FINAL}\rangle & =|0\rangle_{a} \otimes|0\rangle \otimes \ldots \otimes|0\rangle \otimes|\psi\rangle_{b}=\alpha_{0}|00 \ldots 00\rangle+\alpha_{1}|00 \ldots 01\rangle .
\end{aligned}
$$

The main goal of perfect state transfer is to achieve, at some time $t$,

$$
\mid\left\langle\text { FINAL }\left|e^{-i t \mathcal{H}_{G}}\right| \mathrm{START}\right\rangle \mid=1 .
$$

Natural assumptions can be placed on $\mathcal{H}_{G}$ which will allow us to view (3) as a continuous-time quantum walk on $G$.

For example, one typically assumes $\mathcal{H}_{G}$ commutes with $\mathcal{Z}=\sum_{u \in V} Z_{u}$, where the latter operator counts the number of qubits in the $|1\rangle$ state 1 . Note that the eigenvalues of $\mathcal{Z}$ are given by $\lambda_{k}=$ $-n+2 k$, for $k=0,1, \ldots, n$. Since $|00 \ldots 00\rangle$ belongs to the zero eigenspace of $\mathcal{H}_{G}$, we may focus on the unitary evolution of $|10 \ldots 00\rangle$ under $e^{-i t \mathcal{H}_{G}}$. The latter state belongs to the eigenspace $\Lambda_{1}$ of $\mathcal{Z}$ corresponding to the eigenvalue $\lambda_{1}=-n+2$ (since it has exactly one qubit in the $|1\rangle$ state). Thus,

$$
e^{-i t \mathcal{H}_{G}}\left(\alpha_{0}|00 \ldots 00\rangle+\alpha_{1}|10 \ldots 00\rangle\right)=\alpha_{0}|00 \ldots 00\rangle+\alpha_{1} e^{-i t \mathcal{H}_{G}}|10 \ldots 00\rangle .
$$

Since $\mathcal{H}_{G}$ and $\mathcal{Z}$ commute, the eigenspace $\Lambda_{1}$ is $\mathcal{H}_{G}$-invariant; this is because if $|z\rangle$ is an eigenvector of $\mathcal{Z}$ with eigenvalue $\lambda$, then so is $\mathcal{H}_{G}|z\rangle$. Thus, the time evolution of $\exp \left(-i t \mathcal{H}_{G}\right)|10 \ldots 00\rangle$ stays inside the eigenspace $\Lambda_{1}$. Moreover, we have the following basis states for $\Lambda_{1}$ :

$$
|1\rangle=|100 \ldots 0\rangle, \quad|2\rangle=|010 \ldots 0\rangle, \quad \ldots, \quad|n\rangle=|000 \ldots 1\rangle
$$

which forms a natural correspondence with the vertices of $G$; thus, $|a\rangle=|1\rangle$ and $|b\rangle=|n\rangle$. Furthermore, suppose $\mathcal{H}_{G}$ agrees with $A(G)$ on the subspace $\Lambda_{1}$ where $\left\langle v\left|\mathcal{H}_{G}\right| u\right\rangle$ equals the weight $\omega_{u, v}$ of the edge $(u, v)$, for all $u, v \in V$. Examples of $\mathcal{H}_{G}$ satisfying these assumptions include the XY exchange Hamiltonian $\mathcal{H}_{G}=\frac{1}{2} \sum_{(u, v) \in E(G)} \omega_{u, v}\left(X_{u} X_{v}+Y_{u} Y_{v}\right)$, as well as the Heisenberg exchange (which is related to the Laplacian of $G$ ). This shows that the $2^{n}$-dimensional time evolution $e^{-i t \mathcal{H}_{G}}|\mathrm{START}\rangle$ can be viewed as a $n$-dimensional time evolution $e^{-i t A(G)}|a\rangle$ since the former is confined to the single-excitation subspace $\Lambda_{1}$. Further background on these connections may be found in [7, 11, 28, 3].

By the preceding arguments, we may study perfect state tranfer (3) as a continuous-time quantum walk on the graph $G$ (see Farhi and Gutmann [14]). Thus, without loss of generality, we say a graph $G=(V, E)$ has perfect state transfer (PST) from $a$ to $b$ at time $t$ if

$$
\left|\left\langle b\left|e^{-i t A(G)}\right| a\right\rangle\right|=1,
$$

where $A(G)$ is the adjacency matrix of $G$ (thus, focusing on the XY interaction model). This allows us to investigate mathematical properties of the graph $G$ which enable such phenomena to occur. The reduction to quantum walk on graphs was a crucial element in the early works on perfect state transfer (see [7, 1, 12, 11]).

Christandl et al. [12, 11] showed that taking an $k$-fold Cartesian graph product of either a 2path or a 3-path (that is, $K_{2}$ or $P_{3}$ ) with itself yields a high-diameter graph which has perfect state transfer. This follows since both $K_{2}$ and $P_{3}$ have antipodal perfect state transfer and because the Cartesian product operation preserves perfect state transfer. They also made a crucial connection

\footnotetext{
${ }^{1}$ Here, $Z_{u}$ denotes an $n$-fold tensor product of identity matrices except at position $u$ which has the Pauli $Z$ matrix; the same convention applies to the other Pauli matrices.
} 

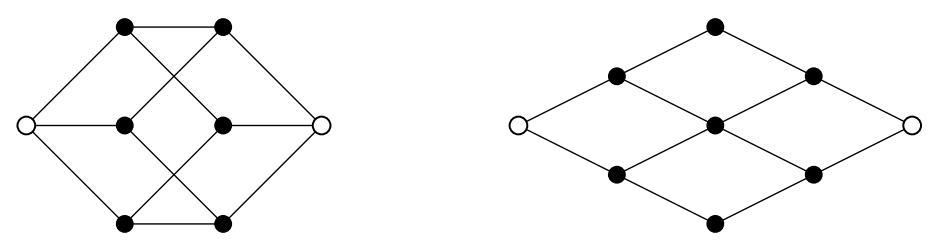

Figure 1: The Cartesian product construction for perfect state transfer (PST): (a) $K_{2}^{\square 3}$; (b) $P_{3}^{\square 2}$ (see Christandl et al. [1]). Antipodal PST occurs between vertices marked white.

between hypercubes and weighted paths using the so-called path-collapsing argument2. Christandl et al. [11] also observed that the $n$-path $P_{n}$, for $n \geq 4$, has no antipodal perfect state transfer but a suitably weighted version of it has perfect state transfer. The weighting scheme on $P_{n}$ is derived from a path-collapsed $(n-1)$-cube.

In an intriguing work, Feder [15] generalized the construction of the weighted paths with perfect state transfer described by Christandl et al. in [12, 11. His construction used a many-boson quantum walk on a single primary graph. He showed that this induced a single-boson quantum walk on a secondary graph and that the secondary graph has perfect state transfer if the primary graph does. In this construction, the weighted path of length $n$ is obtained from a $(n-1)$-boson quantum walk on $K_{2}$.

In algebraic graph theory, the main question is to find a characterization of graphs which exhibit perfect state transfer. Some progress on specific families of graphs were given by Bernasconi et al. [5] and by Cheung and Godsil [8] for hypercubic graphs and by Bašić and Petković [4 for circulant graphs. Although a general characterization remains beyond the reach of current methods, strong general results towards this goal were recently proved by Godsil [18, 19. In one of his results, Godsil proved that a necessary condition for $G$ to have perfect state transfer between vertices $a$ and $b$ is that they are cospectral, that is, the vertex-deleted subgraphs $G \backslash a$ and $G \backslash b$ are isomorphic. This intuitively suggests that the neighborhoods around $a$ and $b$ must look similar. In fact, prior to this work, all known examples of graphs with perfect state transfer between vertices $a$ and $b$ admit an automorphism which maps $a$ to $b$. In [24], Kay proved that the latter property is necessary for paths, while in [18, Godsil asked if this necessary condition holds for any graph.

Our goal in the present work is to explore the role of quotient graphs in perfect state transfer. Since quotient graphs naturally arise in the context of equitable partitions, we use this formalization to capture the idea behind path-collapsing arguments [12, 11, 10]. We argue that equitable partitions provide the most natural way to view these arguments since the resulting proofs are more transparent. Moreover, equitable partitions have been studied extensively in algebraic graph theory (see Godsil and McKay [20] and Godsil and Royle [21]) and have a well-established collection of results which we can build upon.

A main observation we use throughout is the following statement which admits a simple proof: a graph $G$ has perfect state transfer if and only if its quotient graph $G / \pi$ modulo an equitable partition $\pi$ has perfect state transfer. Although weaker forms of this statement had appeared in different guises before, we give a simple and direct proof using the machinery of equitable partitions. The necessary condition was used by Christandl et al. [12, 11] to establish that certain weighted paths have perfect state transfer (in contrast to its unweighted variants). Childs et al. [10] used

\footnotetext{
${ }^{2}$ This argument was used earlier by Childs et al. 10, in the context of exponential algorithmic speedup for a graph search problem.
} 

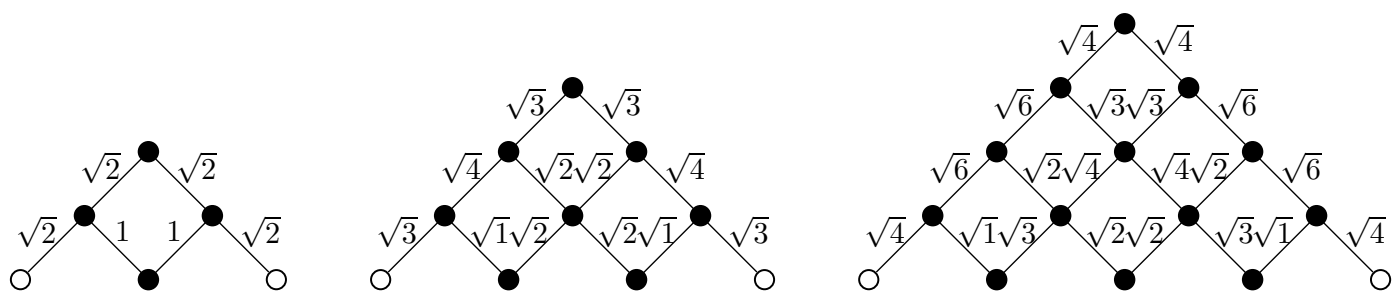

Figure 2: Feder's weighted lattice PST graphs obtained from $k$-boson quantum walks on $P_{3}$ with $k=2,3,4$ (see Feder [15]). Equivalently, these are the quotient graphs $P_{3}^{\square k} / \pi$ (see Theorem 8). Antipodal PST occurs between vertices marked white.

the sufficient condition to analyze hitting times of specific graphs related to binary trees. We will use the backward implication of the equivalence to construct new perfect state transfer graphs. In our first application, we use this lifting property to construct a graph with perfect state transfer between two vertices but has no automorphism which maps one vertex to the other. This answers the aforementioned question posed by Godsil [18].

Using equitable partitions, we also provide an algebraic framework to Feder's construction. We prove that the secondary graph obtained from a $k$-boson quantum walk on a primary graph $G$ is equivalent to a quotient of the $k$-fold Cartesian product of $G$, that is, $G^{\square k} / \pi$, for some equitable partition $\pi$. This equivalence is related to works by Audenaart et al. [3] on symmetric powers of graphs and by Osborne [28] on wedge product on graphs. Our work differs from [3] in that we preserve diagonal entries and from [28] in that we work in a symmetric vector space (rather than exterior vector space). A common thread in all these works is the use of algebraic graph theory to provide an explicit connection between many-particle and single-particle quantum walks. Another related work along the same lines was given in [32]. In our algebraic formalism, we employ a model of many-particle quantum walk used by Gamble et al. [16] and by Smith [29] in their works on graph isomorphism.

Finally, we explore Feder's construction when distinct primary graphs with commensurable perfect state transfer (or even periodic) times are used. We prove a composition theorem which shows partial commutativity between the Cartesian product and quotient operators. This mixed construction is akin to perfect state transfer graphs obtained using weak and lexicographic products (see Ge et al. [17]) and graph joins (see Angeles-Canul et al. [2]). We found new families of perfect state transfer graphs using cube-like graphs (which were studied by Bernasconi et al. [5] and by Cheung and Godsil [8]). The graphs derived from these cube-like graphs are different from weighted graphs obtained in Feder's construction.

Our proofs rely on basic ideas from algebraic graph theory and exploit spectral properties of the underlying graphs.

\section{Preliminaries}

For a logical statement $\mathcal{S}$, the expression $\llbracket \mathcal{S} \rrbracket$ equals 1 if $\mathcal{S}$ is true and 0 otherwise. We use $[n]$ to denote $\{1,2, \ldots, n\}$. The all-one $m \times n$ matrix is denoted by $J_{m, n}$; we also use $\mathbf{j}_{n}$ to denote the all-one $n$-dimensional column vector.

The graph $G=(V, E)$ we study are finite, undirected, and connected. The adjacency matrix $A(G)$ of $G$ is defined as $A(G)_{u, v}=\llbracket(u, v) \in E \rrbracket$. A graph $G$ is called $k$-regular if each vertex 
$u$ of $G$ has exactly $k$ adjacent neighbors. We say a graph $G$ is $(n, k)$-regular if it has $n$ vertices and is $k$-regular. The distance $d(a, b)$ between vertices $a$ and $b$ is the length of the shortest path connecting them. For weighted graphs $G=(V, E, \omega)$, where $\omega: E \rightarrow \mathbb{R}^{+}$is the weight function on edges, we let $A(G)_{u, v}=\omega(u, v)$ be the edge weight of $(u, v)$.

An automorphism $\tau$ of a graph $G=(V, E)$ is a bijective map on the vertex set $V$ that respects the edge relation $E$; that is, $(u, v) \in E$ if and only if $(\tau(u), \tau(v)) \in E$. If $P$ is a permutation matrix which represents an automorphism $\tau$ of $G$, then $P$ commutes with $A(G)$, or $P A(G)=A(G) P$. The automorphism group of $G$ is denoted $\operatorname{Aut}(G)$.

Standard graphs we consider include complete graphs $K_{n}$, paths $P_{n}$, and Cayley graphs. For a given group $\mathcal{G}$ and a subset $S \subseteq \mathcal{G}$, the Cayley graph $X(\mathcal{G}, S)$ has the group $\mathcal{G}$ as its vertex set where two group elements $g$ and $h$ are adjacent if $g h^{-1} \in S$. For $X(\mathcal{G}, S)$ to be connected, we require $S$ to be a generating set of $\mathcal{G}$. If $S$ is closed under taking inverses, that is, $S^{-1}=S$, then $X(\mathcal{G}, S)$ is undirected. An $n$-vertex circulant graph $G$ is a Cayley graph $X\left(\mathbb{Z}_{n}, S\right)$ of the cyclic group of order $n$. Known examples of circulants include complete graphs $K_{n}$ and cycles $C_{n}$.

The complement of a graph $G=(V, E)$, denoted $\bar{G}$, is a graph where $u$ is adjacent to $v$ if and only if $(u, v) \notin E$, for $u \neq v$. The Cartesian product $G \square H$ is a graph defined on the vertex set $V(G) \times V(H)$ where $\left(g_{1}, h_{1}\right)$ is adjacent to $\left(g_{2}, h_{2}\right)$ if either $g_{1}=g_{2}$ and $\left(h_{1}, h_{2}\right) \in E_{H}$, or $\left(g_{1}, g_{2}\right) \in E_{G}$ and $h_{1}=h_{2}$. The adjacency matrix of $G \square H$ is $A(G) \otimes I+I \otimes A(H)$. The $n$ dimensional hypercube (or $n$-cube) $Q_{n}$ is defined recursively as $Q_{1}=K_{2}$ and $Q_{n}=K_{2} \square Q_{n-1}$, for $n \geq 2$. Note $Q_{n}$ is simply the Cayley graph $X\left(\mathbb{Z}_{2}^{n}, S\right)$, where $S$ is the standard generating set for $\mathbb{Z}_{2}^{n}$.

The join $G+H$ is a graph defined on $V(G) \cup V(H)$ obtained by taking two disjoint copies of $G$ and $H$ and by connecting all vertices of $G$ to all vertices of $H$. The cone of a graph $G$ is defined as $K_{1}+G$ whereas the double cone of $G$ is given by $\bar{K}_{2}+G$.

A (vertex) partition $\pi$ of a graph $G=(V, E)$ given by $V=\biguplus_{j=1}^{m} V_{j}$ is called equitable if the number of neighbors in $V_{k}$ of any vertex in $V_{j}$ is a constant $d_{j, k}$, independent of the choice of that vertex (see [21]). We call each component $V_{j}$ a partition or a cell of $\pi$. We say a graph $G$ has an equitable distance partition $\pi$ with respect to vertices $a$ and $b$ if both $a$ and $b$ belong to singleton cells. Further background on algebraic graph theory may be found in the standard monographs by Biggs [6] and by Godsil and Royle [21].

Continuous-time quantum walk For a graph $G=(V, E)$ with adjacency matrix $A(G)$, a continuous-time quantum walk on $G$ is defined through the time-dependent unitary matrix

$$
U_{G}(t)=\exp (-i t A(G)) .
$$

This model was introduced by Farhi and Gutmann [14. We say that $G$ has perfect state transfer (PST) from vertex $a$ to vertex $b$ at time $t$ if

$$
\left|\left\langle b\left|U_{G}(t)\right| a\right\rangle\right|=1
$$

where $|a\rangle,|b\rangle$ denote the unit vectors corresponding to the vertices $a$ and $b$ of $G$, respectively. The graph $G$ has perfect state transfer if there exist vertices $a$ and $b$ in $G$ and time $t$ for which Equation (8) is true. We call a graph $G$ periodic at vertex $a$ if it has perfect state transfer from $a$ to itself at some time $t>0$. Further background on quantum walks and perfect state transfer may be found in the surveys [25, 26] and [24, 18, 30, 27]. 

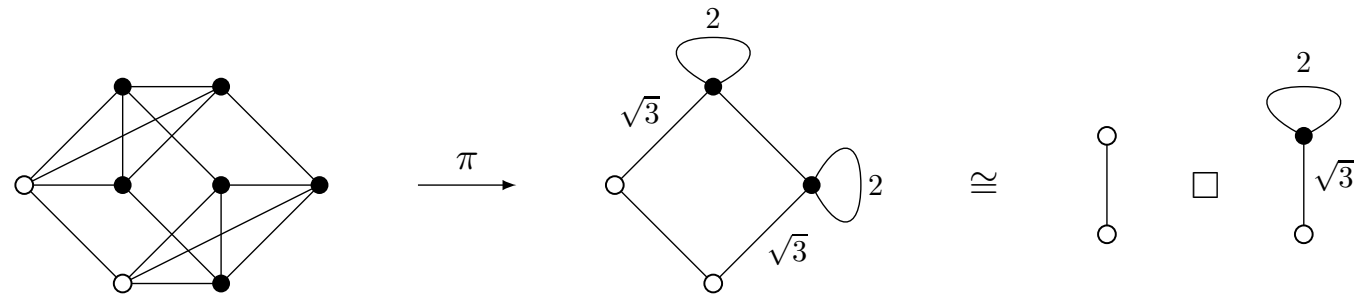

Figure 3: The cube-like graph $X\left(\mathbb{Z}^{3},\{100,010,001,011\}\right)$ (see Bernasconi et al. [5]). Its quotient graph is a Cartesian product of a perfect state transfer and a periodic graph (both at time $\pi / 2$ ).

\section{$3 \quad$ Equitable partitions and quotient graphs}

Christandl et al. [11] showed that certain weighted paths have perfect state transfer by appealing to a path-collapsing argument. Their argument is based on the fact that the $n$-cube $Q_{n}$ has perfect state transfer and it can be collapsed to a weighted path. So they deduce that weighted paths have perfect state transfer since the underlying $n$-cube $Q_{n}$ has this property. This argument was used in the opposite direction by Childs et al. [10] in the context of exponential algorithmic speedup of a quantum walk search algorithm. Here, they deduced properties of the underlying unweighted graphs based on properties of the weighted paths.

A natural way to view this path-collapsing argument is via equitable partitions. The benefit of this is evident in the simple algebraic equivalence of perfect state transfer between a graph and its quotient. The notion of equitable partition was introduced by Godsil and McKay [20] in their work on walk-regular graphs. Our treatment here follows closely the ones given by Godsil and Royle [21] and by Godsil [19, 18].

Let $G=(V, E)$ be a graph with an equitable partition $\pi=\biguplus_{k=1}^{m} V_{k}$ into $m$ cells. For each $j, k \in[m]$, let $d_{j, k}$ be the number of neighbors in $V_{k}$ of any vertex in $V_{j}$ (which is independent of the choice of the vertex). The partition matrix $P$ associated with $\pi$ is defined as the $|V| \times m$ matrix where $P_{x, k}$ equals 1 if vertex $x$ belongs to partition $V_{k}$, and equals 0 otherwise; that is, $P_{x, k}=\llbracket x \in V_{k} \rrbracket$. The quotient graph $G / \pi$ defined in the literature is a weighted directed graph whose adjacency matrix is defined as $B(G / \pi)_{j, k}=d_{j, k}$. A fundamental fact here is that $A(G) P=P B(G / \pi)$ (see [21, Lemma 9.3.1, page 196).

We focus on quotient graphs which are undirected. So, we consider the normalized partition matrix $Q$ defined as

$$
Q=\sum_{k=1}^{m} \frac{1}{\sqrt{\left|V_{k}\right|}} P|k\rangle\langle k| .
$$

Note that $Q_{x, k}=\left|V_{k}\right|^{-1 / 2} P_{x, k}$, and so $Q$ is simply $P$ with each column normalized. Moreover, we still have the fundamental relation $A(G) Q=Q A(G / \pi)$, where $A(G / \pi)$ is a symmetric matrix defined by

$$
A(G / \pi)_{j, k}=\sqrt{d_{j, k} d_{k, j}} .
$$

So, $A(G / \pi)$ describes a weighted graph $G / \pi$ which is an undirected quotient graph of $G$ with respect to the equitable partition $\pi$. We state further useful properties of the partition matrix $Q$.

Lemma 1 (Godsil [19, 18]) The following properties on $Q$ hold:

$$
\text { 1. } Q^{T} Q=I_{m}
$$


2. $Q Q^{T}=\operatorname{diag}\left(\left|V_{k}\right|^{-1} J_{\left|V_{k}\right|}\right)_{k=1}^{m}$.

3. $Q Q^{T}$ commutes with $A(G)$.

4. $A(G / \pi)=Q^{T} A(G) Q$.

The following theorem relates the perfect state transfer properties of a graph $G$ and its quotient $G / \pi$ with respect to an equitable distance partition $\pi$. A similar statement appeared in Ge et al. [17] but our proof here is simpler and more direct.

Theorem 2 Let $G=(V, E)$ be a graph with an equitable partition $\pi$ where vertices a and $b$ belong to singleton cells. Then, for any time $t$

$$
\left\langle b\left|e^{-i t A(G)}\right| a\right\rangle=\left\langle\pi(b)\left|e^{-i t A(G / \pi)}\right| \pi(a)\right\rangle .
$$

Therefore, $G$ has perfect state transfer from a to $b$ at time $t$ if and only if $G / \pi$ has perfect state transfer from $\pi(a)$ to $\pi(b)$ at time $t$.

Proof Since $A(G)$ commutes with $Q Q^{T}$, we have $\left(Q Q^{T} A(G)\right)^{k}=A(G)^{k} Q Q^{T}$ for $k \geq 1$. Given that $a$ and $b$ are in singleton cells, $|\pi(a)\rangle=Q^{T}|a\rangle$ and $|\pi(b)\rangle=Q^{T}|b\rangle$. Thus, we have

$$
\begin{aligned}
\left\langle\pi(b)\left|e^{-i t A(G / \pi)}\right| \pi(a)\right\rangle & =\left\langle\pi(b)\left|e^{-i t Q^{T} A(G) Q}\right| \pi(a)\right\rangle \\
& =\left\langle b\left|Q\left[\sum_{k=0}^{\infty} \frac{(-i t)^{k}}{k !}\left(Q^{T} A(G) Q\right)^{k}\right] Q^{T}\right| a\right\rangle \\
& =\left\langle b\left|\left[\sum_{k=0}^{\infty} \frac{(-i t)^{k}}{k !}\left(Q Q^{T} A(G)\right)^{k}\right] Q Q^{T}\right| a\right\rangle, \quad \text { by regrouping } \\
& =\left\langle b\left|e^{-i t A(G)} Q Q^{T}\right| a\right\rangle,
\end{aligned}
$$

which proves the claim since $Q Q^{T}|a\rangle=|a\rangle$ because $a$ belongs to a singleton cell.

\section{Lifting graph constructions}

In this section, we focus on the backward implication of Theorem 2, This is a lifting theorem which states if a quotient graph $G / \pi_{1}$ has perfect state transfer, for some equitable partition $\pi_{1}$, then the graph $G$ itself must have perfect state transfer. This also implies that any quotient of $G$, say $G / \pi_{2}$, for any other equitable partition $\pi_{2}$, has perfect state transfer. We use this property to construct new graphs with perfect state transfer.

In [18, Godsil asked the following question: if a graph $G$ has perfect state transfer between vertices $a$ and $b$, does there exist an automorphism of $G$ which maps a to b? We contrast this to Kay's notion of a symmetry operator $S$ on $G$ which is a unitary operator satisfying $S A(G)=A(G) S$ and $S|a\rangle=|b\rangle$. In this latter case, Kay [24] proved that such an operator $S$ always exists; but Godsil's question went further and asked if there always exists such an $S$ which is also a graph automorphism of $G$. The question is interesting since, prior to this work, all known graphs with perfect state transfer exhibit this automorphism property.

We answer Godsil's question in the negative by constructing a perfect state transfer graph which lacks the requisite automorphism. Our construction proceeds by lifting a simple weighted 4-vertex path onto a glued double-cone graph. The latter graph was considered earlier in Ge et al. [17] but in a completely different context. We start with a simple observation. 

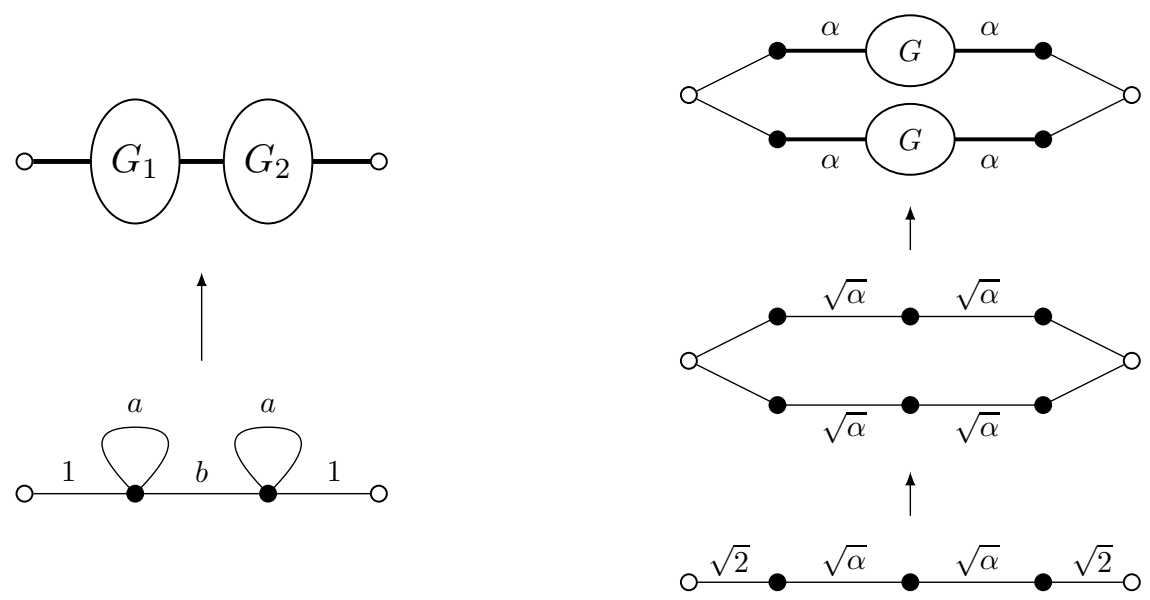

Figure 4: Lifting small PST paths: (i) $\mathscr{P}_{4}(a, b)$ and its lifted graph $K_{1}+G_{1} \circ G_{2}+K_{1}$, where both $G_{1}$ and $G_{2}$ are $(n, a \sqrt{n})$-regular graphs and the connection between them is $(b \sqrt{n})$-regular; $G_{1}$ and $G_{2}$ need not be isomorphic. Here $a=2 k^{2} / \sqrt{4 k^{2}-1}$ and $b=2\left(k^{2}-1\right) / \sqrt{4 k^{2}-1}$, or vice versa, with PST time $t=\pi / 2$. (ii) General weighting on $\mathscr{P}_{5}(\alpha)$ and two of its lifted graphs, where $G$ is the empty graph and $\alpha=4 k^{2}-1, k \geq 1$, with PST time $t=\pi / \sqrt{2}$. Note $k=1$ yields a quotient of the 4-cube $Q_{4}$.

Fact 3 Let $\mathscr{P}_{4}(a, b)$ be a weighted path parametrized by edge-weights a and $b$ (see Figure $4(i)$ ) whose adjacency matrix is:

$$
A=\left[\begin{array}{llll}
0 & 1 & 0 & 0 \\
1 & a & b & 0 \\
0 & b & a & 1 \\
0 & 0 & 1 & 0
\end{array}\right]
$$

Let $\Delta_{ \pm}=\sqrt{\frac{1}{4}(a \pm b)^{2}+1}$. Then, $\mathscr{P}_{4}(a, b)$ has antipodal (vertex 1 to 4 ) perfect state transfer at time $t$ if either

(a) $\cos \left(t \Delta_{+}\right) \cos \left(t \Delta_{-}\right)=+1$ and $\sin (t b / 2)= \pm 1$; or

(b) $\cos \left(t \Delta_{+}\right) \cos \left(t \Delta_{-}\right)=-1$ and $\cos (t b / 2)= \pm 1$.

Proof Let $k_{ \pm}=\frac{1}{2}(a \pm b)$ and $\Delta_{ \pm}^{2}=k_{ \pm}^{2}+1$. The eigenvalues of $\mathscr{P}_{4}(a, b)$ are given by $\alpha_{ \pm}=k_{+} \pm \Delta_{+}$ and $\beta_{ \pm}=k_{-} \pm \Delta_{-}$with the following corresponding eigenvectors

$$
\left|\alpha_{ \pm}\right\rangle=\frac{1}{M_{ \pm}}\left[\begin{array}{llll}
1 & \alpha_{ \pm} & \alpha_{ \pm} & 1
\end{array}\right]^{T}, \quad\left|\beta_{ \pm}\right\rangle=\frac{1}{N_{ \pm}}\left[\begin{array}{llll}
1 & \beta_{ \pm} & -\beta_{ \pm} & -1
\end{array}\right]^{T}
$$

where $M_{ \pm}^{2}=2\left(1+\alpha_{ \pm}^{2}\right)$ and $N_{ \pm}^{2}=2\left(1+\beta_{ \pm}^{2}\right)$ are normalization factors. Assuming the antipodal vertices are $u$ and $v$, we have:

$$
\left\langle v\left|e^{-i t A}\right| u\right\rangle=\sum_{ \pm} \frac{e^{-i t \alpha_{ \pm}}}{M_{ \pm}^{2}}-\sum_{ \pm} \frac{e^{-i t \beta_{ \pm}}}{N_{ \pm}^{2}} .
$$


Since $\left(M_{+} M_{-}\right)^{2}=16 \Delta_{+}^{2}$ and $\left(N_{+} N_{-}\right)^{2}=16 \Delta_{-}^{2}$, we get

$$
\begin{aligned}
& \sum_{ \pm} \frac{e^{-i t \alpha_{ \pm}}}{M_{ \pm}^{2}}=\frac{e^{-i t k_{+}}}{2}\left[\cos \left(t \Delta_{+}\right)+i \frac{k_{+}}{\Delta_{+}} \sin \left(t \Delta_{+}\right)\right] \\
& \sum_{ \pm} \frac{e^{-i t \beta_{ \pm}}}{N_{ \pm}^{2}}=\frac{e^{-i t k_{-}}}{2}\left[\cos \left(t \Delta_{-}\right)+i \frac{k_{-}}{\Delta_{-}} \sin \left(t \Delta_{-}\right)\right] .
\end{aligned}
$$

This proves the claim.

The next theorem shows a construction of a family of graphs with perfect state transfer between antipodal vertices but which has no automorphism exchanging the two vertices.

Theorem 4 For $m \geq 2$, let $n=15 \cdot 2^{2(m-2)}, a=6 \cdot 2^{m-2}$, and $b=8 \cdot 2^{m-2}$. Let $G_{n}$ be the family of graphs of the form $K_{1}+\mathscr{A}_{n} \circ \mathscr{B}_{n}+K_{1}$, where $\mathscr{A}_{n}=\operatorname{Circ}(n,\{ \pm(\lfloor n / 2\rfloor+1), \ldots, \pm(\lfloor n / 2\rfloor+a / 2)\})$ and $\mathscr{B}_{n}=\operatorname{Circ}(n,\{ \pm 1, \ldots, \pm a / 2\})$ are two non-isomorphic families of $n$-vertex a-regular circulant graphs, and the connection $\mathscr{A}_{n} \circ \mathscr{B}_{n}$ is given by a graph $C_{n}$ which is an arbitrary $n$-vertex circulant of degree $b$. Thus, the adjacency matrix of $G_{n}$ is given by:

$$
\left[\begin{array}{cccc}
0 & \mathbf{j}_{n}^{T} & 0 & 0 \\
\mathbf{j}_{n} & \mathscr{A}_{n} & C_{n} & 0 \\
0 & C_{n}^{T} & \mathscr{B}_{n} & \mathbf{j}_{n} \\
0 & 0 & \mathbf{j}_{n}^{T} & 0
\end{array}\right]
$$

Let $a_{n}$ and $b_{n}$ be the antipodal vertices of $G_{n}$. Then $G_{n}$ has perfect state transfer between $a_{n}$ and $b_{n}$ but there is no automorphism $\tau \in \operatorname{Aut}\left(G_{n}\right)$ with $\tau\left(a_{n}\right)=b_{n}$.

Proof The graph $G_{n}$ has a path-like structure with four layers where the two endpoint vertices have degree $n$ each and the middle two "vertices" are $a$-regular graphs (given by $\mathscr{A}_{n}$ and $\mathscr{B}_{n}$ ) which are connected to each other through a $b$-regular structure (given by $C_{n}$ ). Thus, its quotient graph is a weighted $P_{4}$ whose endpoint vertices are connected by edges of weight $\sqrt{n}$ to the middle vertices; and, the two middle vertices have self-loops with weight $a$ each and are connected to each other with an edge of weight $b$; see Figure 4. After normalizing the outer two edges to unit weights, we get $G_{n} / \pi \cong \mathscr{P}_{4}(6 / \sqrt{15}, 8 / \sqrt{15})$, where $\pi$ is the equitable partition where the antipodal vertices belong to singleton cells.

By Fact 3, the quotient graph $G_{n} / \pi$ has antipodal perfect state transfer. Therefore, we know $G_{n}$ has antipodal perfect state transfer by a lifting argument via Theorem 2. It remains to show that the graphs $\mathscr{A}_{n}$ and $\mathscr{B}_{n}$ used to construct $G_{n}$ are nonisomorphic. This holds because $\mathscr{B}_{n}$ contains too many triangles whereas $\mathscr{A}_{n}$ has too few.

A triangle in a circulant $\operatorname{Circ}\left(\mathbb{Z}_{n}, S\right)$ is given by $d_{1}+d_{2}+d_{3} \equiv 0(\bmod n)$ where $d_{1}, d_{2}, d_{3}$ belong to the generating set $S$. It is clear $\mathscr{B}_{n}$ has at least two triangles using $d_{1}=d_{2}= \pm 1$ and $d_{3}=\mp 2$. For $\mathscr{A}_{n}$, we first consider the case when $m>2$ or when $n$ is even. Each generator of $\mathscr{A}_{n}$ is of the form $n / 2 \pm j$, where $j \in\{1, \ldots, a / 2\}$. In this case, $d_{1}+d_{2}+d_{3} \equiv n / 2+\left(j_{1}+j_{2}+j_{3}\right) \not \equiv 0$ $(\bmod n)$, since $j_{1}+j_{2}+j_{3}$ is at most $3 a / 2$ or is at least $-3 a / 2$ and $3 a / 2<n / 2$ by the choice of $n$ and $a$. Finally, if $m=2$, each vertex of $\mathscr{A}_{n}$ is contained in exactly one triangle, by inspection.

Thus, $G_{n}$ has no automorphism which maps $a_{n}$ to $b_{n}$ (the two antipodal vertices of $G_{n}$ ), since otherwise this automorphism will induce an isomorphism between $\mathscr{A}_{n}$ and $\mathscr{B}_{n}$. This is because this automorphism must provide an isomorphism between the neighborhoods of $a_{n}$ and of $b_{n}-$ which in our example are simply the graphs $\mathscr{A}_{n}$ and $\mathscr{B}_{n}$, respectively. This is impossible since $\mathscr{A}_{n}$ 

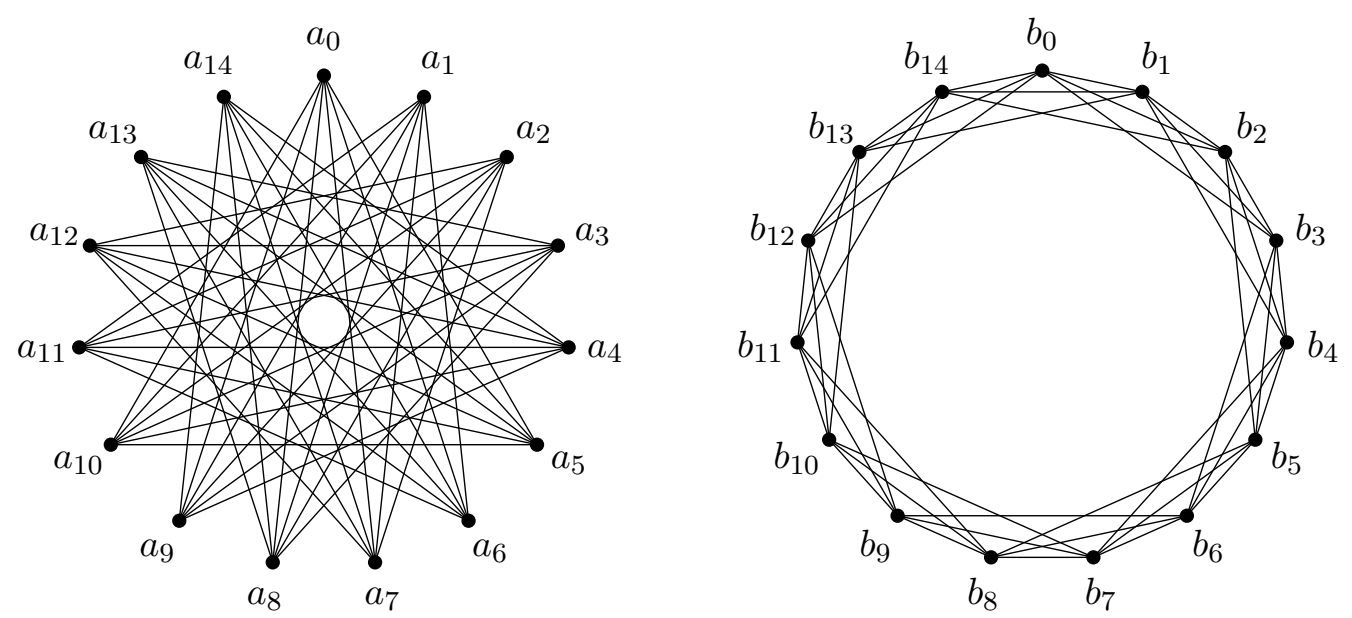

Figure 5: The graphs $\mathscr{A}_{2}$ and $\mathscr{B}_{2}$ used for $K_{1}+\mathscr{A}_{2} \circ \mathscr{B}_{2}+K_{1}$ in Theorem 4 ,

and $\mathscr{B}_{n}$ are non-isomorphic.

Our lifting technique can be applied to other families of small weighted paths.

Fact 5 Let $\mathscr{P}_{5}(a, b)$ be a weighted path (see Figure 4(ii)) whose adjacency matrix is:

$$
A=\left[\begin{array}{lllll}
0 & a & 0 & 0 & 0 \\
a & 0 & b & 0 & 0 \\
0 & b & 0 & b & 0 \\
0 & 0 & b & 0 & a \\
0 & 0 & 0 & a & 0
\end{array}\right]
$$

Let $\Delta=a \sqrt{1+b^{2}}$. Then, $\mathscr{P}_{5}(a, b)$ has antipodal perfect state transfer at time $t$ if $a=\sqrt{2}$, $\cos (a t)=-1$ and $\cos (\Delta t)=1$. Moreover, these conditions hold with $b=\sqrt{4 k^{2}-1}$, for $k \geq 1$.

Proof The eigenvalues of $A$ are $0, \pm a$ and $\pm \Delta$ with the following corresponding eigenvectors:

$$
\begin{aligned}
& |0\rangle=\frac{1}{\sqrt{2\left(1+1 / b^{2}\right)}}\left[\begin{array}{lllll}
1 & 0 & -a / b & 0 & 1
\end{array}\right]^{T} \\
& \left|a_{ \pm}\right\rangle=\frac{1}{2}\left[\begin{array}{lllll}
\mp 1 & -1 & 0 & +1 & \pm 1
\end{array}\right]^{T} \\
& \left|\Delta_{ \pm}\right\rangle=\frac{1}{2 \Delta / a}\left[\begin{array}{lllll}
1 & \pm \Delta / a & a b & \pm \Delta / a & 1
\end{array}\right]^{T}
\end{aligned}
$$

The choice of $a=\sqrt{2}$ is determined by the eigenvector form of $\left|\Delta_{ \pm}\right\rangle$. We leave $a$ as a variable whenever possible but use $a=\sqrt{2}$ if it leads to simpler expressions. If the antipodal vertices are denoted $u$ and $v$, we have

$$
\left\langle v\left|e^{-i t A}\right| u\right\rangle=\frac{b^{2}}{2\left(1+b^{2}\right)}-\frac{\cos (a t)}{2}+\frac{\cos (\Delta t)}{2\left(1+b^{2}\right)}=\frac{b^{2}+\cos (\Delta t)}{2\left(b^{2}+1\right)}-\frac{\cos (a t)}{2} .
$$


To get perfect state transfer from $u$ to $v$, it suffices to require $\cos (\Delta t)=1$ and $\cos (a t)=-1$. Since $a=\sqrt{2}$, we have $t=\pi / \sqrt{2}$. The condition $\cos (\Delta t)=1$ with $b=\sqrt{4 k^{2}-1}$ and $t=\pi / \sqrt{2}$ is equivalent to $\cos (2 \pi k)=1$, which holds for any $k \geq 1$.

Remark: Fact 5 shows that $\mathscr{P}_{5}\left(\sqrt{2}, \sqrt{4 k^{2}-1}\right)$, where $k \geq 1$, is a family of perfect state transfer paths whose first member $\mathscr{P}_{5}(\sqrt{2}, \sqrt{3})$ is simply the quotient of the cube $Q_{4}=K_{2}^{\square 4}$. Figure 4 shows an example of two lifted graphs obtained from this family.

\section{Quotient graph constructions}

Feder [15] described an intriguing construction of perfect state transfer graphs using many-boson quantum walks. First, we review the basic ideas of this construction, and then we describe its algebraic characterization using quotient graphs.

Let $G=(V, E)$ be a graph with perfect state transfer which we will call the primary graph. For a positive integer $k$, consider a process of $k$ bosons performing a quantum walk on $G$. A configuration of these $k$ bosons is given by a collection of numbers $\left\{n_{v}: v \in V\right\}$, where $n_{v}$ represents the number of bosons located at vertex $v$, with $0 \leq n_{v} \leq k$. The sum of these numbers must be $k$, that is, $\sum_{v \in V} n_{v}=k$, since there is exactly $k$ bosons at all times. So, a natural choice of basis states for the configurations of the $k$-boson quantum walk is $\left|n_{u_{1}}, n_{u_{2}}, \ldots, n_{u_{n}}\right\rangle$, where $V=\left\{u_{1}, \ldots, u_{n}\right\}$ is the vertex set of $G$. The set of these basis states forms a vertex set in a so-called secondary graph.

In [15], Feder used a nearest-neighbor hopping Hamiltonian $\mathcal{H}=\sum_{(u, v)} a_{u}^{\dagger} a_{v}$, where $a_{u}$ and $a_{u}^{\dagger}$ are the bosonic annihilation and creation operators. The interaction term between the two basis states $\left|n_{u}, n_{v}, n_{W}\right\rangle$ and $\left|n_{u}-1, n_{v}+1, n_{W}\right\rangle$ is $\sqrt{n_{u}\left(n_{v}+1\right)}$, where $n_{u} \geq 1$ and $W=V \backslash\{u, v\}$. We summarize this construction in the following.

Definition 1 (Feder's graph [15]) Let $G=(V, E)$ be a graph and let $k \geq 1$ be a positive integer. Let $\mathcal{G}=(\mathcal{V}, \mathcal{E}, \omega)$ be a weighted graph whose vertex set $\mathcal{V}$ is the basis states $\left|n_{V}\right\rangle=\bigotimes\left\{\left|n_{u}\right\rangle: \sum_{u} n_{u}=k\right\}$ and whose edge set $\mathcal{E}$ is the weighted pairs $\omega\left(\left|n_{u}, n_{v}, n_{W}\right\rangle,\left|n_{u}-1, n_{v}+1, n_{W}\right\rangle\right)=\sqrt{n_{u}\left(n_{v}+1\right)}$, assuming $n_{u} \geq 1$, where $W=V \backslash\{u, v\}$. We call $\mathcal{G}$ the secondary graph of $G$ with $k$ bosons, denoted by $G^{\odot k}$.

A nice property of Feder's construction is that it generalizes the weighted paths of Christandl et al. [12, 11]. As noted earlier, the latter is based on a path-collapsing argument of the $n$-cube. This yields a weighted path $\mathscr{P}_{n+1}$ on the vertex set $\{0,1, \ldots, n\}$ where the edge weight of $(j, j+1)$ is $\sqrt{(j+1)(n-j)}$. In Feder's notation, we have $\mathscr{P}_{n+1}=K_{2}{ }^{\odot n}$. By recursion, this generated various infinite families of graphs with perfect state transfer, with connections to high-dimensional Platonic solids, such as parallelepipeds, hypertetrahedra, hyperoctahedra (see [15]).

Algebraic characterization Our aim in this section is to cast Feder's construction in an algebraic framework. Here, we adopt the explicit many-boson quantum walk model used by Gamble et al. [16] and Smith [29]. The Hamiltonian of the $k$-boson quantum walk in this model is given by

$$
H_{k B}=-\left[\frac{1}{k !} \sum_{\sigma \in S_{k}} P_{\alpha}\right] A\left(G^{\square k}\right),
$$

where $S_{k}$ is the symmetric group of all permutations on $k$ elements. Each permutation $\sigma \in S_{k}$ induces the following natural group action on the elements of $V^{k}$,

$$
\sigma \circ\left(x_{1}, x_{2}, \ldots, x_{k}\right)=\left(x_{\sigma(1)}, x_{\sigma(2)}, \ldots, x_{\sigma(k)}\right) .
$$



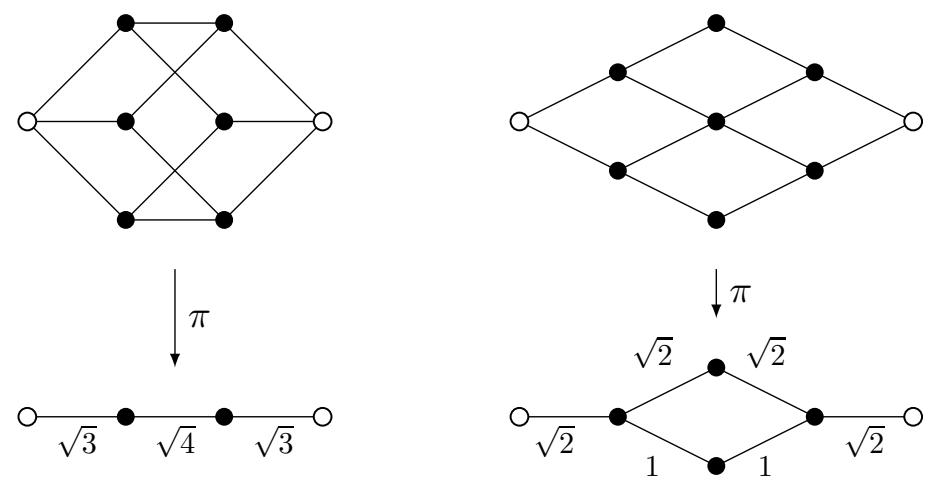

Figure 6: The Cartesian product graphs $K_{2}^{\square 3}$ and $P_{3}^{\square 2}$ and their quotients under equitable partitions, whose cells are orbits of $S_{3}$ and $S_{2}$ acting on the respective vertex sets.

We denote the latter simply as $\sigma(x)$, whenever $x=\left(x_{1}, \ldots, x_{k}\right)$. So, the permutation matrix $P_{\sigma}$ is an $|V|^{k} \times|V|^{k}$ matrix defined as:

$$
\left\langle x\left|P_{\sigma}\right| y\right\rangle=\llbracket y=\sigma(x) \rrbracket .
$$

The time evolution of the $k$-boson quantum walk is then given by $U_{k B}=e^{-i t H_{k B}}$. This description captures the intuition that each boson is performing a quantum walk on its own copy of the graph $G$ but collectively they are performing a quantum walk on $G^{\square k}$. Next, we show that the symmetrization operator in Equation (27) induces an equitable partition on $G^{\square k}$.

Lemma 6 Let $G=(V, E)$ be a graph and $k \geq 1$ be an integer. Then, the operator

$$
\mathbb{S}=\frac{1}{k !} \sum_{\sigma \in S_{k}} P_{\sigma}
$$

which acts on the set $V^{k}$, defines an equitable partition $\pi$ of $G^{\square k}$. Moreover, $\mathbb{S}$ equals $Q Q^{T}$, where $Q$ is the normalized partition matrix of $\pi$.

Remark: Osborne 28] considered a related operator which includes an alternating permutation sign in the summation. Both operators correspond to symmetrization or skew-symmetrization in a symmetric or exterior vector spaces, respectively (see [13], page 452).

Proof Consider a vertex partition $\pi=\biguplus_{x} \mathcal{O}_{x}$ of the product graph $G^{\square k}$ defined by the cells

$$
\mathcal{O}_{x}=\left\{y \in V^{k}: \exists \sigma \in S_{k}, \sigma(x)=y\right\} .
$$

Each cell $\mathcal{O}_{x}$ is an orbit of $S_{k}$ acting on the vertex set $V\left(G^{\square k}\right)=V^{k}$. To show $\pi$ is equitable, let $x$ and $y$ be adjacent vertices in $G^{\square k}$. This implies that there is a unique index $i$ for which $x_{i}$ is adjacent to $y_{i}$ in $G$ and $x_{j}=y_{j}$ for all other $j \neq i$ (this can be shown using induction on $k$ ). Now, let $S$ be the collection of indices where $x_{i}$ appears in $x$; note $i \in S$. Consider a permutation $\tau$ which swaps $i$ with $j \in S \backslash\{i\}$. Then, $x$ is also adjacent to $\tau(y)$; moreover, $\tau(y) \in \mathcal{O}_{y}$. Thus, $x$ has $|S|$ neighbors in $\mathcal{O}_{y}$. Since $x$ is an arbitrary element of $\mathcal{O}_{x}$, every element in $\mathcal{O}_{x}$ has $|S|$ neighbors in $\mathcal{O}_{y}$. This shows $\pi$ is equitable. 


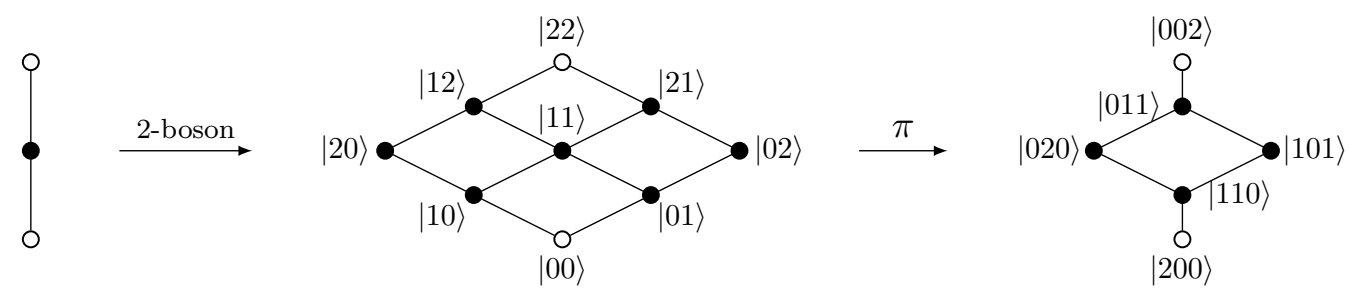

Figure 7: The 2-boson walk on $P_{3}$, its Cartesian product representation $P_{3}^{\square 2}$, and the Feder diamond graph $D_{6}=P_{3} \odot 2 \cong P_{3}^{\square 2} / \pi$. Antipodal PST occur throughout between vertices marked white.

From the definition of $P_{\sigma}$ in Equation (29), we have:

$$
\left\langle x\left|\left[\frac{1}{k !} \sum_{\sigma} P_{\sigma}\right]\right| y\right\rangle=\frac{1}{k !} \sum_{\sigma} \llbracket y=\sigma(x) \rrbracket=\frac{1}{k !}|\operatorname{Stab}(x)| \llbracket y \in \mathcal{O}_{x} \rrbracket=\frac{1}{\left|\mathcal{O}_{x}\right|} \llbracket y \in \mathcal{O}_{x} \rrbracket
$$

where $\operatorname{Stab}(x)=\left\{\sigma \in S_{k}: \sigma(x)=x\right\}$ is the stabilizer of $x$, which is the set of permutations which fix $x$. The last equality follows from $\left|\mathcal{O}_{x}\right||\operatorname{Stab}(x)|=k$ !, since the size of the orbit of $x$ is the index of the stabilizer subgroup of $x$ (see Hungerford [22], Theorem 4.3, page 89). On the other hand, the partition matrix $Q$ of $\pi$ is defined as $\langle x|Q| j\rangle=\left|V_{j}\right|^{-1 / 2} \llbracket x \in V_{j} \rrbracket$. Thus, we have

$$
\left\langle x\left|Q Q^{T}\right| y\right\rangle=\frac{1}{\left|\mathcal{O}_{x}\right|} \llbracket y \in \mathcal{O}_{x} \rrbracket .
$$

This proves our second claim that $\mathbb{S}=Q Q^{T}$.

By Lemma 6, the unitary evolution $U_{k B}$ of the $k$-boson quantum walk admits a simpler description.

Lemma 7 (see [29]) The unitary evolution of the $k$-boson quantum walk on a graph $G$ using the Hamiltonian $H_{k B}=-\left[\frac{1}{k !} \sum_{\sigma \in S_{k}} P_{\alpha}\right] A\left(G^{\square k}\right)$, is given by

$$
U_{k B}=\left[\frac{1}{k !} \sum_{\sigma} P_{\sigma}\right]\left(e^{i t A(G)}\right)^{\otimes k} .
$$

Proof First, we note that $\left(\frac{1}{d} J_{d}\right)^{m}=\frac{1}{d} J_{d}$, for any $d, m \geq 1$. Let $\mathbb{S}=\frac{1}{k !} \sum_{\sigma} P_{\sigma}$ be the "symmetrizing" operator defined in Equation (30). By Lemma 1 and Lemma 6, we have $\mathbb{S}=Q Q^{T}$ and it is a block diagonal matrix containing all-one submatrices. The block diagonal property of $\mathbb{S}$ implies that $\mathbb{S}^{m}=\mathbb{S}$, for any $m \geq 1$. Moreover, again by Lemma 1 , $\mathbb{S}$ commutes with $A\left(G^{\square k}\right)$. Therefore,

$$
\begin{aligned}
U_{k B} & =\exp \left(-i t H_{k B}\right)=\exp \left(i t \mathbb{S} A\left(G^{\square k}\right)\right) \\
& =\sum_{m=0}^{\infty} \frac{(i t)^{m}}{m !} \mathbb{S}^{m} A\left(G^{\square k}\right)^{m}, \quad \text { since } \mathbb{S} \text { commutes with } A\left(G^{\square k}\right) \\
& =\mathbb{S} \exp \left(i t A\left(G^{\square k}\right)\right), \quad \text { since } \mathbb{S}^{m}=\mathbb{S}, \text { for } m \geq 1
\end{aligned}
$$

This proves the claim since $\exp \left(i t A\left(G^{\square k}\right)\right)=\left(e^{i t A(G)}\right)^{\otimes k}$.

The next theorem describes our main algebraic characterization of Feder's construction. We show that the graph $G^{\odot k}$ is a quotient graph of the $k$-fold Cartesian product $G^{\square k}$. Moreover, it shows if $G$ has perfect state transfer, then so does $G^{\odot k}$, which follows immediately from Theorem 2 , 
Theorem 8 Let $G=(V, E)$ be a graph and $k$ be a positive integer. Then,

$$
G^{\odot k} \cong G^{\square k} / \pi
$$

where $\pi$ is an equitable partition of $G^{\square k}$ defined by the cells $\mathcal{O}_{x}=\left\{y: \exists \sigma \in S_{k}, \sigma(x)=y\right\}$. Moreover, if $G$ has perfect state transfer then so does $G^{\odot k}$, for any positive integer $k$.

Proof Let $\mathcal{G}=(\mathcal{V}, \mathcal{E})$ be the graph $G^{\odot k}$ described in Definition 1, where $\mathcal{V}$ is the set of $|V|$ dimensional vectors whose entries are non-negative integers that sum to $k$. For $x \in V^{k}$, let $n[x]$ be a $|V|$-dimensional vector whose $u$-th entry, for $u \in V$, is given by

$$
n[x]_{u}=\left|\left\{i \in[k]: x_{i}=u\right\}\right|
$$

which is the number of occurrences of vertex $u$ in $x$. Consider the map $\phi: V\left(G^{\square k}\right) \rightarrow \mathcal{V}$ defined by $\phi(x)=n[x]$. By definition of $\mathcal{O}_{x}$, we have $n[y]=n[x]$ for all $y \in \mathcal{O}_{x}$. So, we may view $\phi$ as a mapping from $V\left(G^{\square k} / \pi\right)$ to $\mathcal{V}$.

Next, we show that $\phi$ is a graph isomorphism between the quotient graph $G^{\square k} / \pi$ and Feder's graph $\mathcal{G}$. Consider two vertices $\mathcal{O}_{x}$ and $\mathcal{O}_{y}$ of the quotient graph $G^{\square k} / \pi$ whose edge weight between them is $\sqrt{d_{x, y} d_{y, x}}$. Here, $d_{x, y}$ is the number of neighbors in $\mathcal{O}_{y}$ that each vertex in $\mathcal{O}_{x}$ has and $d_{y, x}$ is the number of neighbors in $\mathcal{O}_{x}$ that each vertex in $\mathcal{O}_{y}$ has.

Let $\phi\left(\mathcal{O}_{x}\right)=n[x]$ and $\phi\left(\mathcal{O}_{y}\right)=n[y]$. If $x$ and $y$ are adjacent in the product graph $G^{\square k}$, then $x$ and $y$ differ in exactly one coordinate $i$, where $x_{i}$ and $y_{i}$ are adjacent in $G$, and agree in the other coordinates. Suppose $x_{i}=u$ and $y_{i}=v$ with $u \neq v$ but $u$ is adjacent to $v$ in $G$. Then, $n[y]_{u}=n[x]_{u}-1$ and $n[y]_{v}=n[x]_{v}+1$. By Definition 1, the edge weight between $n[x]$ and $n[y]$ in $\mathcal{G}$ is given by

$$
\omega(n[x], n[y])=\sqrt{n[x]_{u}\left(n[x]_{v}+1\right)}
$$

which equals to

$$
\omega\left(\mathcal{O}_{x}, \mathcal{O}_{y}\right)=\sqrt{d_{x, y} d_{y, x}}
$$

since $d_{x, y}=n[x]_{u}$ (the number of ways to replace $u$ with $v$ ) and $d_{y, x}=n[y]_{v}+1$ (the number of ways to replace $v$ with $u$ ). This shows that $G^{\odot k} \cong G^{\square k} / \pi$.

The next theorem shows a composition theorem for Feder's operator $G^{\odot k}$. We will use this to describe a reduction method from one perfect state transfer graph to another by combining and alternating lifting and quotient operations.

Theorem 9 For a given graph $G$ and integers $m_{1}, m_{2} \geq 1$, let $\pi_{1}$ be an equitable partition of $G^{\square m_{1}}$ and let $\pi_{2}$ be an equitable partition of $\left(G^{\square m_{1}} / \pi_{1}\right)^{\square m_{2}}$. Then, there is an equitable partition $\pi_{3}$ of $G^{\square\left(m_{1} m_{2}\right)}$ where

$$
\left(G^{\square m_{1}} / \pi_{1}\right)^{\square m_{2}} / \pi_{2} \cong G^{\square\left(m_{1} m_{2}\right)} / \pi_{3} .
$$

Proof Let $Q_{1}$ and $Q_{2}$ be the (normalized) partition matrices corresponding to $\pi_{1}$ and $\pi_{2}$, respectively. The adjacency matrix of $\left(G^{\square m_{1}} / \pi_{1}\right)^{\square m_{2}}$ is given by

$$
\sum_{k=1}^{m_{2}}(I \otimes \ldots \otimes \overbrace{Q_{1}^{T} A\left(G^{\square m_{1}}\right) Q_{1}}^{k \text {-th position }} \otimes \ldots I),
$$




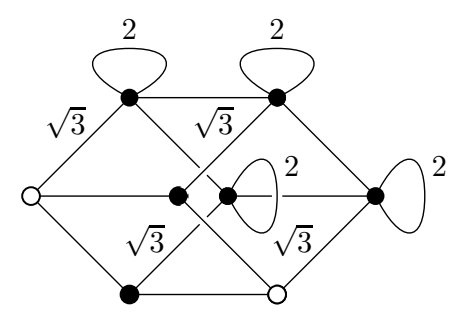

Figure 8: The perfect state transfer graph $K_{2} \square\left(X\left(\mathbb{Z}_{2}^{3},\{001,010,100,011\}\right) / \pi\right)$, where the latter is a Cartesian product of $K_{2}$ with a periodic graph.

since $A\left(G^{\square m_{1}} / \pi_{1}\right)=Q_{1}^{T} A\left(G^{\square m_{1}}\right) Q_{1}$. By expressing the identity matrices as $Q_{1}^{T} Q_{1}$ and factoring it out from both sides, we get

$$
\left(Q_{1}^{T}\right)^{\otimes m_{2}}\left[\sum_{k=1}^{m_{2}}(I \otimes \ldots \otimes \overbrace{A\left(G^{\square m_{1}}\right)}^{k \text {-th position }} \otimes \ldots I)\right] Q_{1}^{\otimes m_{2}} .
$$

The last equation yields

$$
\left(Q_{1}^{\otimes m_{2}}\right)^{T}\left[A\left(G^{\square m_{1}}\right)^{\square m_{2}}\right] Q_{1}^{\otimes m_{2}}=\left(Q_{1}^{\otimes m_{2}}\right)^{T} A\left(G^{\square\left(m_{1} m_{2}\right)}\right) Q_{1}^{\otimes m_{2}} .
$$

Thus, the adjacency matrix of $\left(G^{\square m_{1}} / \pi_{1}\right)^{\square m_{2}} / \pi_{2}$ is given by

$$
Q_{2}^{T}\left(Q_{1}^{\otimes m_{2}}\right)^{T} A\left(G^{\square\left(m_{1} m_{2}\right)}\right) Q_{1}^{\otimes m_{2}} Q_{2},
$$

which proves the claim and shows $\pi_{3}$ is defined by the partition matrix $Q_{1}^{\otimes m_{2}} Q_{2}$.

Remark: Using Theorem 9, the perfect state transfer graphs described in [15] arguably are all quotients of the $n$-cube derived using different equitable partitions. For example, the graph shown in Figure 7 is derived from the 4 -cube since $P_{3}^{\square 2} / \pi_{1} \cong\left(K_{2}^{\square 2} / \pi_{2}\right)^{\square 2} / \pi_{1} \cong K_{2}^{\square 4} / \pi_{3}$.

\section{Generalizations}

\subsection{Inhomogeneous products}

Note that Feder's construction is based on taking the Cartesian product of a single perfect state transfer graph with itself followied by a quotient operation. Here, we extend this construction by using distinct perfect state transfer and periodic graphs in the product and by allowing the quotient operations to alternate with the product. But first, we show a composition theorem for this more general construction (similar to Theorem 9).

Theorem 10 For $n \in \mathbb{N}$ and for each $k \in[n]$, let $G_{k}$ be a graph and $\pi_{k}$ be an associated equitable partition. Then, there is an equitable partition $\pi$ so that

$$
\square_{k=1}^{n}\left(G_{k} / \pi_{k}\right)=\left(\square_{k=1}^{n} G_{k}\right) / \pi .
$$

Moreover, if $Q_{k}$ is the partition matrix of $\pi_{k}$, then $\bigotimes_{k=1}^{n} Q_{k}$ is the partition matrix of $\pi$. 
Proof Let $Q_{k}$ be the normalized partition matrix of $\pi_{k}$. The adjacency matrix of $G_{k} / \pi_{k}$ is defined by $Q_{k}^{T} A\left(G_{k}\right) Q_{k}$. Thus, the adjacency matrix of $\square_{k}\left(G_{k} / \pi_{k}\right)$ is

$$
\sum_{k=1}^{n}(I \otimes \ldots \otimes \overbrace{Q_{k}^{T} A\left(G_{k}\right) Q_{k}}^{k \text {-th position }} \otimes \ldots \otimes I) .
$$

Now, replace each $I$ in the term above by $Q_{j}^{T} Q_{j}$ if it is in position $j \neq k$. This gives us

$$
\sum_{k=1}^{n}(Q_{1}^{T} Q_{1} \otimes \ldots \otimes \overbrace{Q_{k}^{T} A\left(G_{k}\right) Q_{k}}^{k \text {-th position }} \otimes \ldots \otimes Q_{n}^{T} Q_{n}) .
$$

Factoring the common terms $Q_{k}^{T}$ on the left and $Q_{k}$ on the right, we get

$$
\left(\bigotimes_{k=1}^{n} Q_{k}^{T}\right) \sum_{k=1}^{n}(I \otimes \ldots \otimes \overbrace{A\left(G_{k}\right)}^{k \text {-th position }} \otimes \ldots \otimes I)\left(\bigotimes_{k=1}^{n} Q_{k}\right) .
$$

This yields

$$
\left(\bigotimes_{k=1}^{n} Q_{k}\right)^{T} A\left(\square_{k=1}^{n} G_{k}\right)\left(\bigotimes_{k=1}^{n} Q_{k}\right) .
$$

which shows that $Q=\bigotimes_{k=1}^{n} Q_{k}$ is the partition matrix of $\pi$.

The following corollary extends Feder's operator $G^{\odot k}$ which is based on a single graph $G$. Here, we take a product of different graphs $G_{k}$ (and their quotients $G_{k} / \pi_{k}$ ) and allow both perfect state transfer and periodic graphs with commensurable times.

Corollary 11 Let $n \geq 1$ be an integer. For $k \in[n]$, let $G_{k}$ be a graph with perfect state transfer between vertices $a_{k}$ and $b_{k}$ at time $t\left(G_{k}\right.$ is periodic, if $\left.a_{k}=b_{k}\right)$, where $a_{k} \neq b_{k}$ for at least one $k$. Let $\pi_{k}$ be an equitable distance partition of $G_{k}$ with respect to $a_{k}$ and $b_{k}$. Then

$$
\square_{k=1}^{n}\left(G_{k} / \pi_{k}\right) \cong\left(\square_{k=1}^{n} G_{k}\right) / \pi
$$

has perfect state transfer between $\left(a_{1}, \ldots, a_{n}\right)$ and $\left(b_{1}, \ldots, b_{n}\right)$ at time $t$. Here, $\pi$ is an equitable partition of $\square_{k} G_{k}$ defined by the partition matrix $\otimes_{k} Q_{k}$, where $Q_{k}$ is the partition matrix of $\pi_{k}$.

We show an example of how to build new perfect state transfer graphs using Corollary 11, For this, we use the following powerful results on cube-like graphs proved by Bernasconi et al. [5] and by Cheung and Godsil [8].

Theorem 12 (Bernasconi et al. [5] and Cheung-Godsil [8])

Let $G=X\left(\mathbb{Z}_{2}^{n}, S\right)$ be the Cayley graph on $\mathbb{Z}_{2}^{n}$ with generating set $S$ and let $\omega_{S}=\sum_{a \in S}$ a be the sum of the elements in $S$. Let $M$ be the $n \times|S|$ matrix with elements of $S$ as columns and whose row space is called the code of $G$. Also, let $D$ be the greatest common divisor of the weights of the codewords of $G$. Then, the following holds:

1. If $\omega_{S} \neq 0$, then $G$ has perfect state transfer from 0 to $\omega_{S}$ at time $t=\pi / 2$. 

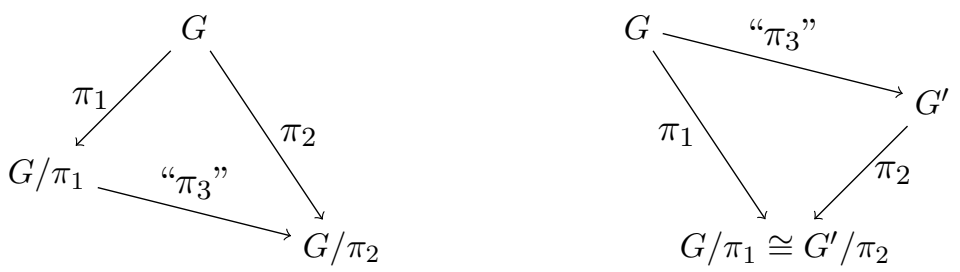

Figure 9: (i) Lift-and-quotient: if $G / \pi_{1}$ has PST, then $G$ has PST; which implies $G / \pi_{2}$ has PST. So, $G / \pi_{1}$ reduces to $G / \pi_{2}$ via " $\pi_{3}$ ". (ii) Quotient-and-lift: if $G$ has PST, then $G / \pi_{1}$ has PST; which implies $G^{\prime}$ has PST if $G^{\prime} / \pi_{2} \cong G / \pi_{1}$. So, $G$ reduces to $G^{\prime}$ via " $\pi_{3}$ ".

2. If $\omega_{S}=0$, then $G$ has perfect state transfer at time $t=\pi / 4$ if and only if $D=2$ and the code of $G$ is self-orthogonal.

Remark: Let $G_{k}=X\left(\mathbb{Z}_{2}^{n}, S_{k}\right)$ be any collection of cube-like graphs defined in Theorem 12, where at least one satisfies $\sum_{a \in S_{k}} a \neq 0$. This guarantees that at least one graphs has "antipodal" perfect state transfer at time $\pi / 2$, while the others might be periodic at time $\pi / 2$. By Corollary 11, we know $\square_{k}\left(G_{k} / \pi_{k}\right) \cong\left(\square_{k} G_{k}\right) / \pi$ has perfect state transfer, for any collection of equitable partitions $\left\{\pi_{k}\right\}$. A simple example of this construction is given in Figure 8 ,

\subsection{Reductions}

In this section, we describe reductions between perfect state transfer graphs obtained from alternating a lifting move (from a quotient graph $G / \pi$ to a graph $G$, for an equitable partition $\pi$ ) and a quotient move (from the graph $G$ to its quotient graph $G / \pi$, for a possibly different equitable partition). By interchanging the order of these two operations, we get a quotient-and-lift reduction or a lift-and-quotient reductions. We illustrate these two types of reductions in Figure 9 .

As a simple example, consider the diamond graph $D_{6}$ from Figure 7 , There is a lift-and-quotient reduction from $D_{6}$ to $\mathscr{P}_{5}$ given by

$$
D_{6} \nearrow G \searrow \mathscr{P}_{5}
$$

where $G$ is the graph obtained from attaching two vertices onto $K_{2,3}$ (each with edge weight $\sqrt{2}$ ). This reduction is depicted in Figure 10. Note we get PST on $G$ for "free". An alternate lift-andquotient reduction based on Theorem 9 is given by

$$
D_{6}=P_{3}^{\square 2} / \pi_{1} \nearrow\left(K_{2}^{\square 2} / \pi_{2}\right)^{\square 2} / \pi_{1} \cong K_{2}^{\square 4} / \pi_{3} \searrow \mathscr{P}_{5}
$$

where here $G$ is the 4-cube $Q_{4}=K_{2}^{\square 4}$.

Irreducible graphs Godsil's question in [18] is closely related to an observation of Kay [23] that any weighted path with perfect state transfer must have mirror-symmetric weights. Given the construction described in Section 4, it is natural to ask if there is a class of graphs for which perfect state transfer implies the automorphism property. Let $G=(V, E)$ be a graph with perfect state transfer between vertices $a$ and $b$. For each vertex $x \in V$, let $d_{a}(x)$ (respectively, $d_{b}(x)$ ) be the distance of $x$ from $a$ (respectively, $b$ ). To each vertex $x$, we assign the distance-pair $d_{a, b}(x)=$ $\left(d_{a}(x), d_{b}(x)\right)$ of $x$ from both $a$ and $b$. We say $G$ is distance-minimal with respect to vertices $a$ and $b$ if each vertex has a unique distance-pair, that is, for $x \neq y$, we have $d_{a, b}(x) \neq d_{a, b}(y)$. Alternatively, 


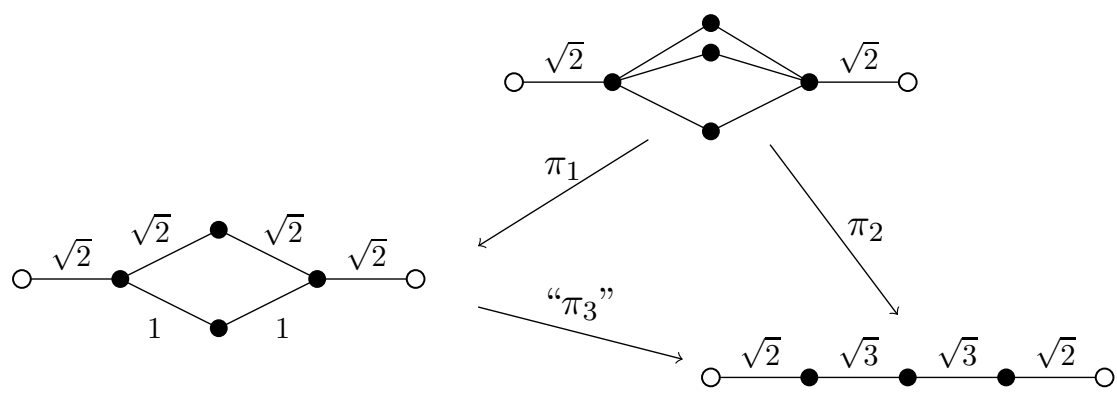

Figure 10: The lift-and-quotient reduction: $D_{6}=P_{3}^{\square 2} / \pi$ is lifted to the top graph $G$ (via the "inverse" of $\pi_{1}$ ) whose "other" quotient is $D_{6} / \pi_{3}$. We infer $G$ has PST for "free".

we say a graph $G_{1}$ is reducible to $G_{2}$ (with respect to vertices $a$ and $b$ ) if there is a lift-and-quotient or a quotient-and-lift reduction from $G_{1}$ to $G_{2}$ which places vertices $a$ and $b$ in singleton cells, so that $G_{2}$ has fewer vertices than $G_{1}$. We call a graph quotient-minimal if it is not reducible to any other graph. Let us call a graph minimal if it is either distance-minimal or quotient-minimal. Intuitively, if a graph is minimal, it can only have (if any) an automorphism switching $a$ and $b$ since the action of permuting vertices at the same distance from $a$ or $b$ have been ruled out.

Conjecture 1 Let $G$ be a graph with perfect state transfer between vertices a and $b$. If $G$ is minimal with respect to $a$ and $b$, then $G$ has an automorphism $\tau \in \operatorname{Aut}(G)$ so that $\tau(a)=b$.

\section{Conclusions}

In this work, we explored perfect state transfer in quantum walks using equitable partitions. Our main focus is on a strong equivalence of perfect state transfer between a graph and its quotients. Although weaker forms of this equivalence had appeared earlier, we gave a simple and most direct proof which yields a powerful two-way tool (taking lifts and quotients on graphs) to study perfect state transfer.

In lifting, if a perfect state transfer graph is a quotient of another graph, then the parent graph also has perfect state transfer. We used this to construct graphs with perfect state transfer between two vertices but which lack automorphism swapping the vertices; hence, answering a question posed by Godsil in [18. This question is relevant since, prior to this work, all known graphs with perfect state transfer admit the automorphism property.

In a quotient move, if a graph has perfect state transfer graph, then so does its quotient. These quotient graphs are obtained by forming various equitable partitions of the original graph. We used this to describe Feder's intriguing construction of PST graphs [15] based on many-boson quantum walks. By adopting an explicit model of $k$-boson quantum walk in [16, 29], we show that Feder's graphs are quotients of a $k$-fold Cartesian product of PST graphs. The resulting graphs have perfect state transfer due to the equivalence theorem. This is related to works by Audenaart [3], by Osborne [28, and by Wieśniak and Markiewicz [32] which used algebraic graph theory to provide explicit connection between multiple and single excitation subspaces under various coupling schemes on graphs. 
It would be interesting to find a property of graphs, for which any graph perfect state transfer graph with this property must admit an automorphism swapping the two perfect state transfer vertices. We leave this as an open question for future work.

\section{Acknowledgments}

The research was supported in part by the National Science Foundation grant DMS-1004531 and also by the National Security Agency grant H98230-11-1-0206. We thank David Feder for kindly describing his construction in [15], Chris Godsil for very helpful comments on quantum walks, Dani ben-Avraham for discussions on bosons, and the anonymous reviewers for constructive comments which improved the presentation of this paper.

\section{References}

[1] C. Albanese, M. Christandl, N. Datta, and A. Ekert. Mirror inversion of quantum states in linear registers. Physical Review Letters, 93:230502, 2004.

[2] R.J. Angeles-Canul, R. Norton, M. Opperman, C. Paribello, M. Russell, and C. Tamon. Perfect state transfer, integral circulants and join of graphs. Quantum Information and Computation, 10(3\&4):325-342, 2010.

[3] K. Audenaart, C. Godsil, G. Royle, and T. Rudolph. Symmetric squares of graphs. Journal of Combinatorial Theory B, 97:74-90, 2007.

[4] M. Bašić and M. Petković. Some classes of integral circulant graphs either allowing or not allowing perfect state transfer. Applied Mathematics Letters, 22(10):1609-1615, 2009.

[5] A. Bernasconi, C. Godsil, and S. Severini. Quantum networks on cubelike graphs. Physical Review A, 78:052320, 2008.

[6] N. Biggs. Algebraic Graph Theory. Cambridge University Press, second edition, 1993.

[7] S. Bose. Quantum communication through an unmodulated spin chain. Physical Review Letters, 91(20):207901, 2003.

[8] W.-C. Cheung and C. Godsil. Perfect state transfer on cubelike graphs. Linear Algebra and Its Applications, 435(10):2468-2474, 2011.

[9] A. Childs. Universal computation by quantum walk. Physical Review Letters, 102:180501, 2009.

[10] A. Childs, R. Cleve, E. Deotto, E. Farhi, S. Gutmann, and D. Spielman. Exponential algorithmic speedup by a quantum walk. In Proc. 35th ACM Symp. Theory of Computing, pages $59-68,2003$.

[11] M. Christandl, N. Datta, T. Dorlas, A. Ekert, A. Kay, and A. Landahl. Perfect transfer of arbitrary states in quantum spin networks. Physical Review A, 71:032312, 2005.

[12] M. Christandl, N. Datta, A. Ekert, and A. Landahl. Perfect state transfer in quantum spin networks. Physical Review Letters, 92:187902, 2004. 
[13] D. Dummit and R. Foote. Abstract Algebra. John Wiley, 2004.

[14] E. Farhi and S. Gutmann. Quantum computation and decision trees. Physical Review A, 58:915-928, 1998.

[15] D. Feder. Perfect quantum state transfer with spinor bosons on weighted graphs. Physical Review Letters, 97:180502, 2006.

[16] J.K. Gamble, M. Friesen, D. Zhou, R. Joynt, and S.N. Coppersmith. Two-particle quantum walks applied to the graph isomorphism problem. Physical Review A, 81(5):052313, 2010.

[17] Y. Ge, B. Greenberg, O. Perez, and C. Tamon. Perfect state transfer, graph products and equitable partitions. International Journal on Quantum Information, 9(3):823-842, 2011.

[18] C. Godsil. State transfer on graphs. Discrete Mathematics 312(1):129-147, 2011.

[19] C. Godsil. When can perfect state transfer occur? quant-ph/1011.0231.

[20] C. Godsil and B. McKay. Feasibility conditions for the existence of walk-regular graphs. Linear Algebra and Its Applications, 30:51-61, 1980.

[21] C. Godsil and G. Royle. Algebraic Graph Theory. Springer, 2001.

[22] T. Hungerford. Algebra. Springer-Verlag, 1974.

[23] A. Kay. Perfect, efficient, state transfer and its applications as a constructive tool. International Journal on Quantum Information, 8(4):641-676, 2009. quant-ph/0903.4274.

[24] A. Kay. Basics of perfect communication through quantum networks. Physical Review A, 84:022337, 2011.

[25] J. Kempe. Quantum random walks - an introductory overview. Contemporary Physics, 44(4):307-327, 2003.

[26] V. M. Kendon. Quantum walks on general graphs. International Journal on Quantum Information, 4(5):791-805, 2006.

[27] V. M. Kendon and C. Tamon. Perfect state transfer in quantum walks on graphs. Journal of Computational and Theoretical Nanoscience, 8(3):422-433, 2011.

[28] T. Osborne. Statics and dynamics of quantum XY and Heisenberg systems on graphs. Physical Review B, 74:094411, 2006.

[29] J. Smith. $k$-boson quantum walks do not distinguish arbitrary graphs. quant-ph/1004.0206.

[30] D. Stevanović. Applications of graph spectra in quantum physics. In D. Cvetković and I. Gutman, editors, Selected Topics in Applications of Graph Spectra, pages 85-111. Mathematical Institute SANU, 2011.

[31] M. Underwood and D. Feder. Universal quantum computation by discontinuous quantum walk. Physical Review A, 82:042304, 2010.

[32] M. Wieśniak and M. Markiewicz. Finding traps in non-linear spin arrays. quant-ph/0911.3579. 